Supporting Information 


\section{Scalable Electrically Conductive Spray Coating Based on Block \\ Copolymer Nanocomposites}

Junpyo Kwon ${ }^{1,2}$, Katherine Evans ${ }^{2,3}$, Le Ma ${ }^{2,4}$, Daniel Arnold ${ }^{5, \dagger}$, M. Erden Yildizdag ${ }^{1, \dagger}$, Tarek Zohdi ${ }^{1}$, Robert O. Ritchie ${ }^{1,2,4}$ and Ting $\mathrm{Xu}^{2,3,4, *}$

${ }^{1}$ Department of Mechanical Engineering, University of California, Berkeley, CA 94720, USA

${ }^{2}$ Materials Sciences Division, Lawrence Berkeley National Laboratory, Berkeley, CA 94720, USA

${ }^{3}$ Department of Chemistry, University of California, Berkeley, CA 94720, USA

${ }^{4}$ Department of Materials Science and Engineering, University of California, Berkeley, CA 94720, USA

${ }^{5}$ Department of Chemical Engineering, University of California, Berkeley, CA 94720, USA

†The present addresses were given under Author Information.

*Correspondence to: tingxu@berkeley.edu

\section{SUPPORTING INFORMATION}

1. Figure $\mathrm{S} 1$

2. Figure $\mathrm{S} 2$

3. Figure $\mathrm{S} 3$

4. Figure S4

5. Supplementary Note 1: Mass fractal dimension, $\boldsymbol{d}_{\boldsymbol{m}}$

6. Supplementary Note: Size of the nano-caviations

7. Supplementary Reference 
a
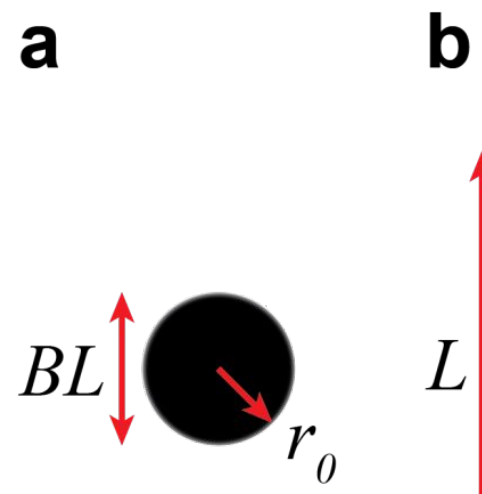

b

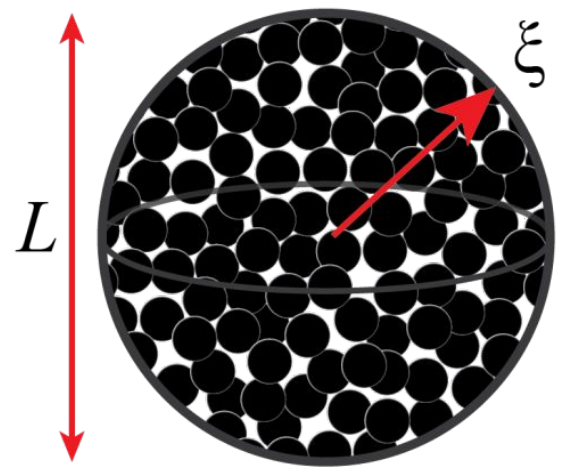

C

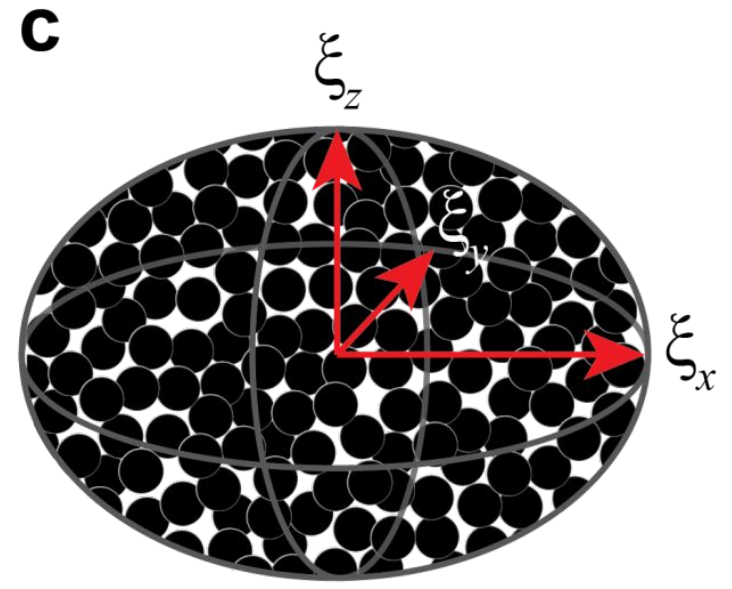

Figure S1. Schematic drawings of (a) the carbon black object, (b) the cluster, and (c) the elliptically deformed cluster. 


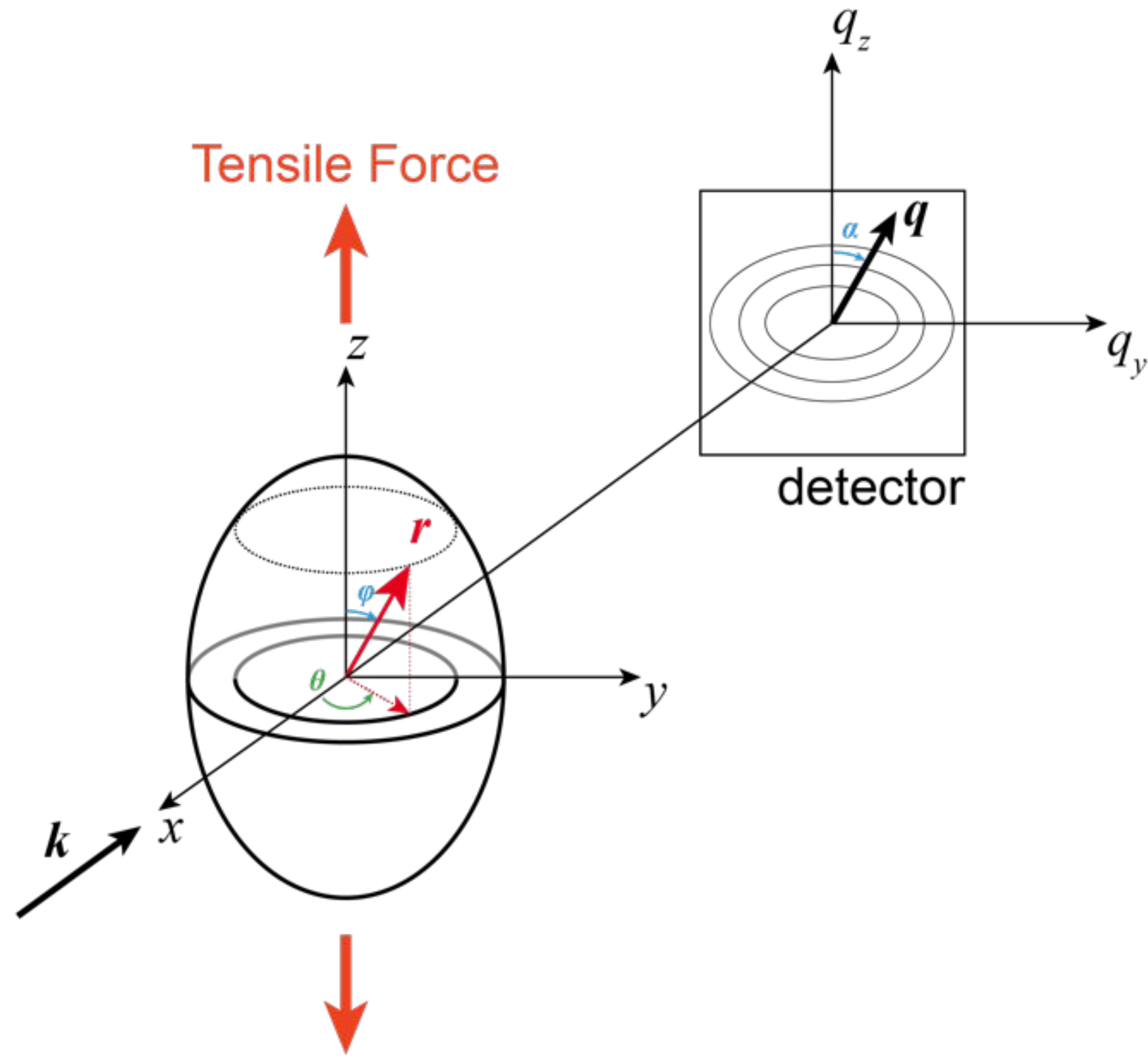

Figure S2. Spherical coordinates used for the derivations of the correlation function of an infinitely extended structure. 


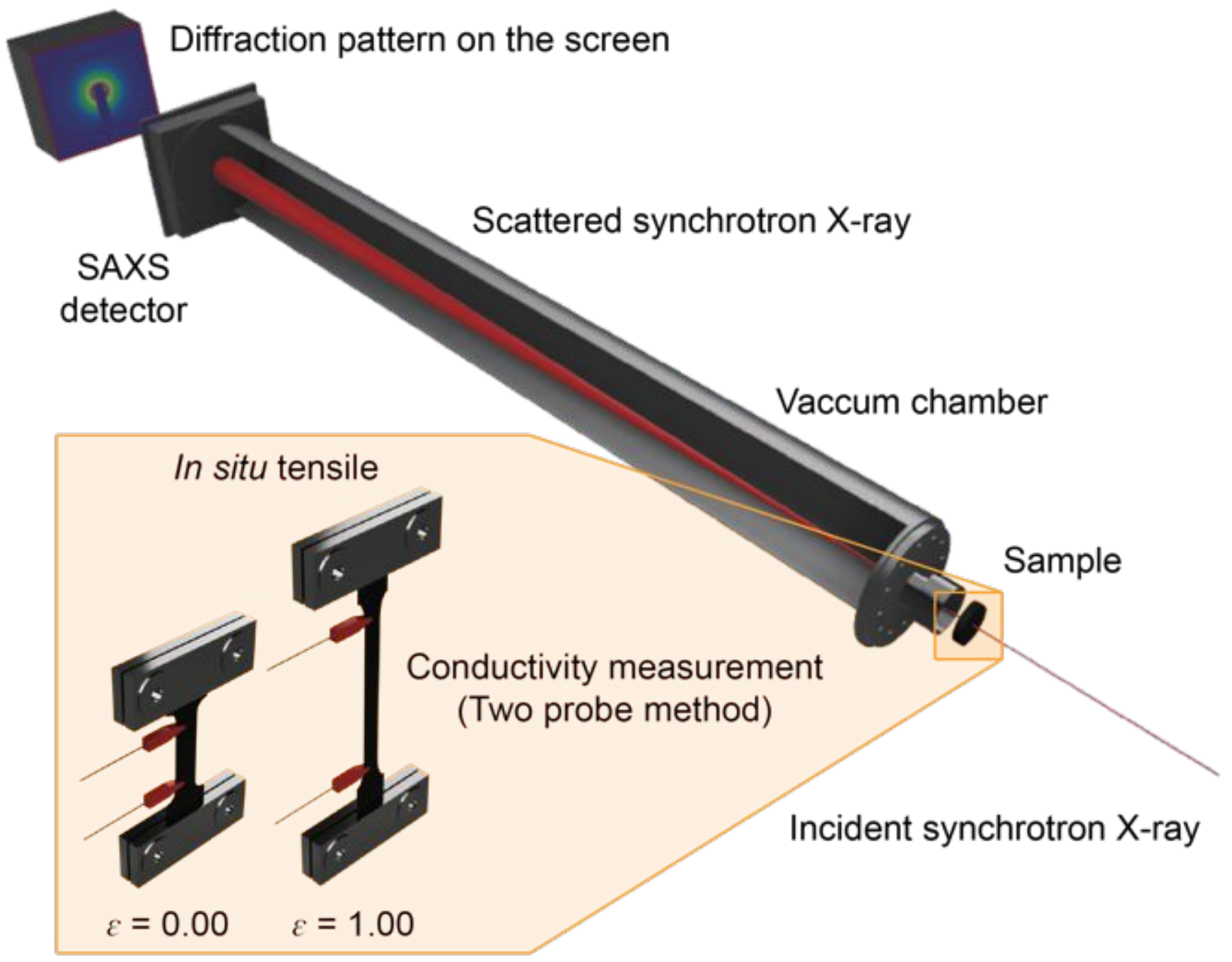

Figure S3. Schematic drawing of the experimental set-up for the synchrotron SAXS during in situ uniaxial tensile testing and measuring the conductivity. 

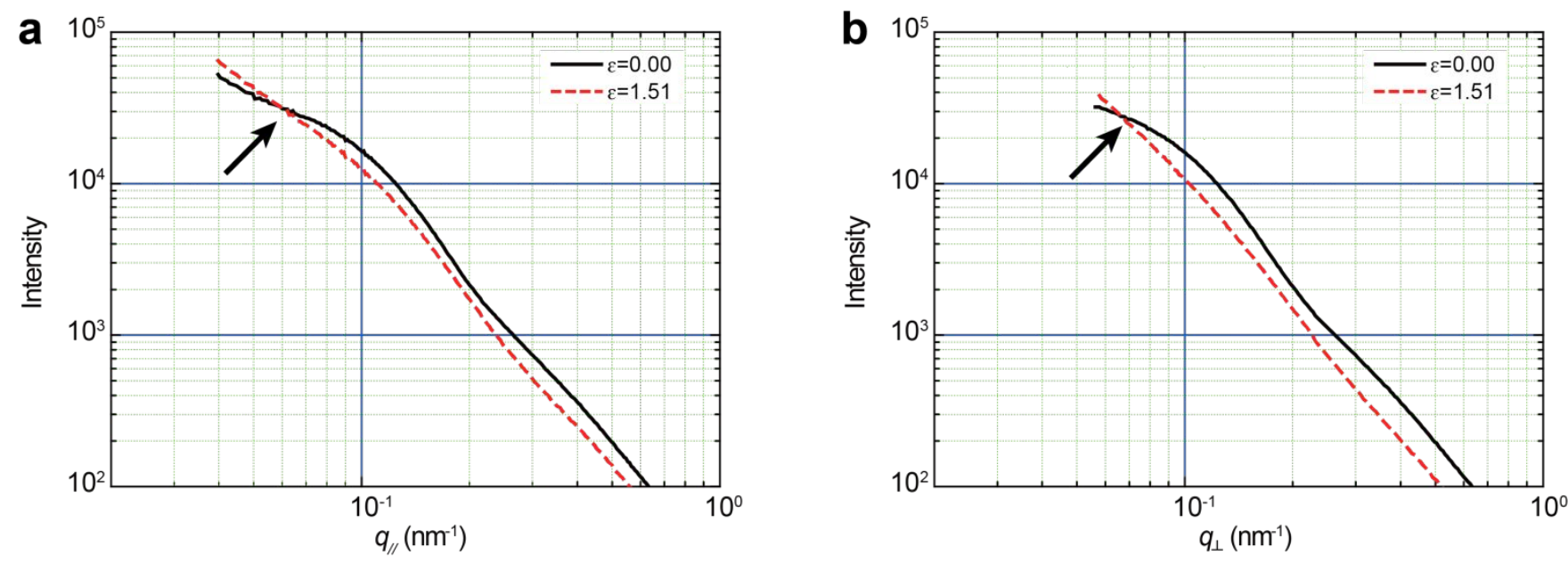

Figure S4. Intensity I vs. $q$ log-log plots from SAXS results along (a) parallel and (b) perpendicular directions to the tensile axis. The black arrows indicate the crossover point of the initial and "butterfly-to-lemon" shaped SAXS spectra transition (see text). 


\section{Supplementary Note 1: Mass fractal dimension, $d_{m}$}

The structure factor of the carbon black fractal aggregates affects the scattered intensity $I(q)$ changes in the power-law domain represented in a $\log -\log$ plot. ${ }^{1}$ The mass fractal dimension $d_{m}$ is the corresponding exponent value, and the dimension can be used to measure how fractal aggregates interpenetrate each other. ${ }^{2}$ Here, we explain how the slope in the Porod regime can represent the mass fractal dimension for the self-similar fractals. For further details, the work of Schneider et al. ${ }^{3-5}$ should be referred to.

The carbon black aggregates are the unit for large carbon black clusters (Figure S1). If the object is picked out from the cluster, the mass $m$ of the object is given by:

$$
m(B L)=B^{d_{0}} m(L),
$$

where $L$ is the size of the cluster, $B$ is the scale factor, and $d_{0}$ is the dimension of the object. Since the object is smaller than the cluster, the value of $B$ should be less than unity; accordingly, Eq. (1) can be simplified as:

$$
m=A L^{d_{0}} .
$$

In reality, the object could exhibit a nonhomogeneous density. Therefore, $d_{0}$ should be replaced with non-integer dimension, $d_{\mathrm{m}}$ :

$$
m=A^{\prime}\left(\frac{\xi}{r_{0}}\right)^{d_{m}} .
$$

Here, $d_{\mathrm{m}}$ represents the distribution of mass within the cluster, which is the mass fractal dimension. $\xi$ and $r_{0}$ are the radius of the cluster and the object, respectively. It should be assumed that the objects are isotropic in order to derive Eq. (3). If we relate mass to the number $N$ of objects in the cluster, then:

$$
N=A\left(\frac{\xi}{r_{0}}\right)^{d_{m}},
$$

where the prefactor $A$ is called the lacunarity. We can assume $A \approx 1$ for simple calculations. For the clusters of carbon black particles, the deformation of the clusters is applied only along a single direction (for uniaxial tensile tests); the clusters are accordingly rescaled in one direction, but not in the others. Therefore, we can regard the carbon black clusters as a self-affine system. Under this system, $N$ can be expressed in three directions in cartesian coordinates as: 


$$
N=\left(\frac{\xi_{x}}{r_{0}}\right)^{d_{x}}=\left(\frac{\xi_{y}}{r_{0}}\right)^{d_{y}}=\left(\frac{\xi_{z}}{r_{0}}\right)^{d_{z}}
$$

$\xi_{x}, \xi_{y}$, and $\xi_{z}$ are the half lengths of the minor and semi-axis of an elliptically deformed carbon black cluster; $d_{x}, d_{y}$, and $d_{z}$ are the directional dependent fractal exponents. Under the assumptions that there are no breaking and/or loosing of the objects during the deformation of the clusters, $N$ remains a constant, and Eq. (5) can be re-written as:

$$
N=\left\{\frac{\left(\xi_{x} \xi_{y} \xi_{z}\right)^{\frac{1}{3}}}{r_{0}}\right\}^{d}
$$

Assuming the deformation is symmetric $\left(\lambda_{x}=\lambda_{y}\right)$, we can define $\lambda_{x}=\xi_{x} / \xi$ and $\lambda_{y}=\xi_{y} / \xi$, where $\lambda$ is the microscopic deformation ratio. Therefore, Eq. (6) can be expressed as:

$$
N=\left(\frac{\xi}{r_{0}}\right)^{d}\left\{\left(\lambda_{x} \lambda_{y} \lambda_{z}\right)^{\frac{1}{3}}\right\}^{d}
$$

Accordingly, based on the assumption of symmetric deformation $\left(\lambda_{x}=\lambda_{y}\right)$, i.e., Eqs. (4) and (7), the relationship between the fractal exponent $d$ and the mass fractal dimension $d_{m}$ can be represented as:

$$
d=d_{m} \frac{1}{1+\left(\frac{1}{3}\right)\left\{\frac{\log \left(\frac{\lambda_{y}^{2}}{\lambda_{z}}\right)}{\log \left(\frac{\xi}{r_{0}}\right)}\right)} .
$$

As we assumed that the objects are isotropically distributed in the cluster, the scattered intensity, $I$, can be separated into the intensity of the particles $P$ and that of the cluster (or structure) $S$ :

$$
I\left(q, r_{0}, \xi, d_{m}\right)=P\left(q, r_{0}\right) S\left(q, r_{0}, \xi, d_{m}\right),
$$

where $P$ and $S$ are defined as the particle and structure factor, respectively. $S$ can be analytically expressed as:

$$
S=1+\int_{\boldsymbol{R}^{3}} \tilde{g}(\boldsymbol{r}) \exp (i \boldsymbol{q} \cdot \boldsymbol{r}) d V
$$

where $\tilde{g}(\boldsymbol{r})$ is the correlation function, which in turn is defined as:

$$
\tilde{g}(\boldsymbol{r})=g(\boldsymbol{r}) \cdot K(\boldsymbol{r})
$$


Here, $g(\boldsymbol{r})$ is the correlation function of an infinitely extended structure (the structure function), $K(\boldsymbol{r})$ incorporates the inhomogeneity and the finite size of the objects (the shape function), and $r$ can be expressed as:

$$
\boldsymbol{r}=\left\{\begin{array}{l}
x \\
y \\
z
\end{array}\right\}=\left\{\begin{array}{c}
\lambda_{y} r \sin \varphi \cos \theta \\
\lambda_{y} r \sin \varphi \cos \theta \\
\lambda_{z} r \cos \varphi
\end{array}\right\}
$$

Coordinate transformation from the cartesian to spherical coordinates is helpful here (Figure S2). In transformed spherical coordinates $(0<r<\infty, 0<\theta<\pi, 0<\varphi<2 \pi)$, the volume element can be derived as:

$$
d V=\lambda_{x} \lambda_{y} \lambda_{z} r^{2} \sin \varphi d \varphi d \theta d r
$$

The scattering vector $q$ can also be written as:

$$
\boldsymbol{q}=\left\{\begin{array}{c}
0 \\
q \sin \alpha \\
q \cos \alpha
\end{array}\right\}
$$

Therefore,

$$
\boldsymbol{q} \cdot \boldsymbol{r}=q r\left(\lambda_{y} \sin \varphi \sin \theta \sin \alpha+\lambda_{z} \cos \varphi \cos \alpha\right)
$$

Under the assumption of symmetric deformation $\left(\lambda_{x}=\lambda_{y}\right)$, Eq. (7) can be re-written as:

$$
N=\left(\frac{r}{r_{0}}\right)^{d}\left\{\left(\lambda_{y}^{2} \lambda_{z}\right)^{\frac{1}{3}}\right\}^{d},
$$

where the derivative of $N$ with respect to $r$ is given as:

$$
\frac{d N}{d r}=d \frac{r^{d-1}}{r_{0}^{d}}\left\{\left(\lambda_{y}^{2} \lambda_{z}\right)^{\frac{1}{3}}\right\}^{d} .
$$

Using the definition of $g(\boldsymbol{r})$, the number $N$ of the objects within the volume $V$ is therefore:

$$
\begin{gathered}
N=\int_{V} g(\boldsymbol{r}) d^{3} r=4 \pi \lambda_{y}^{2} \lambda_{z} \int_{0}^{R} d r r^{2} g(r), \\
d N=4 \pi \lambda_{y}^{2} \lambda_{z} r^{2} g(r) d r .
\end{gathered}
$$

Accordingly, Eqs. (17) and (19) can be combined to describe $g(r)$ as: 


$$
g(r)=d \frac{\left(\lambda_{y}^{2} \lambda_{z}\right)^{\frac{d}{3}-1}}{4 \pi r_{0}^{d}} r^{d-3}
$$

The shape function $K(\boldsymbol{r})$ can be written by applying an exponent function to represent the elliptical shape:

$$
K=\exp \left\{-\left(\frac{x^{2}}{\xi_{x}^{2}}+\frac{y^{2}}{\xi_{y}^{2}}+\frac{z^{2}}{\xi_{z}^{2}}\right)^{\frac{1}{2}}\right\}=\exp \left(-\frac{r}{\xi}\right)
$$

Based on Eqs. (10), (11), (20), and (21), the structure factor $S$ can be analytically expressed as: ${ }^{6}$

$$
\begin{aligned}
S\left(q, r_{0}, \xi, d_{m}\right)= & +\left(\lambda_{y}^{2} \lambda_{z}\right)^{\frac{d}{3}} \frac{\Gamma(d+1)}{\left(\tilde{g} r_{0}\right)^{d}}\left[1+\frac{1}{(\tilde{g} \xi)^{2}}\right]^{\frac{1-d}{2}} \\
& \times \frac{\sin [(d-1) \arctan (\tilde{g} \xi)]}{(d-1)}
\end{aligned}
$$

with the gamma function $\Gamma$ and the abbreviation:

$$
\tilde{q}=q\left(\lambda_{y}^{2} \sin ^{2} \alpha+\lambda_{z}^{2} \cos ^{2} \alpha\right)^{\frac{1}{2}} .
$$

Assuming $\tilde{q} r_{0} \ll 1$ and $\tilde{q} \xi \gg 1$ :

$$
\lim _{\tilde{q} \rightarrow \infty}[S(\tilde{q})-1] \propto \tilde{q}^{-d} .
$$

Therefore, as for self-similar fractals $\left(\lambda_{y}=\lambda_{z}\right)$, the slope can be used to represent the mass fractal dimension $d_{m}$, since $d$ becomes $d_{m}$ based on Eq. (8):

$$
I \propto q^{-d_{m}} .
$$

\section{Supplementary Note 2: Size of the nano-cavitations}

The size of the nano-cavitations can be measured from the small-angle x-ray scattering (SAXS) results. The measured spectra from the scattered phase was shifted from a "butterfly-shaped" to a "lemonshaped" pattern at a tensile strain of roughly $\epsilon \sim 1.51$. The position of the crossover point in parallel and perpendicular directions along the tensile axis provides a means to measure the size of the nanocavitations in these respective directions. ${ }^{7}$ In the parallel direction (where the scattering vector $q \sim 0.6$ 
$\mathrm{nm}^{-1}$ ) and in a perpendicular direction (where $q \sim 0.7 \mathrm{~nm}^{-1}$ ), the width and height of the nano-cavitations can be calculated as $105 \mathrm{~nm}$ and $90 \mathrm{~nm}$, as the real space distance $d=2 \pi / q$ (Figure $\mathrm{S} 4$ ).

\section{AUTHOR INFORMATION}

Corresponding author

Email: tingxu@berkeley.edu

\section{Supplementary References}

(1) Ehrburger-Dolle, F.; Bley, F.; Geissler, E.; Livet, F.; Morfin, I.; Rochas, C. Filler Networks in Elastomers. Macromol. Symp. 2003, 200 (1), 157-168.

(2) Ehrburger-Dolle, F.; Hindermann-Bischoff, M.; Livet, F.; Bley, F.; Rochas, C.; Geissler, E. Anisotropic Ultra-Small-Angle X-Ray Scattering in Carbon Black Filled Polymers. Langmuir 2001, 17 (2), 329-334.

(3) Schneider, G. J. Correlation of Mass Fractal Dimension and Asymmetry. J. Chem. Phys 2009, 130 (23), 234912.

(4) Schneider, G. J.; Goritz, D. A Novel Model for the Interpretation of Small-Angle Scattering Experiments of Self-Affine Structures. J. Appl. Crystallogr. 2010, 43 (1), 12-16.

(5) Schneider, G. J.; Göritz, D. Strain Induced Anisotropies in Silica Polydimethylsiloxane Composites. J. Chem. Phys. 2010, 133 (2), 024903.

(6) Schneider, G. J.; Göritz, D. A Novel Model for the Interpretation of Small-Angle Scattering Experiments of Self-Affine Structures. J. Appl. Crystallogr. 2010, 43 (1), 12-16.

(7) Zhang, H.; Scholz, A. K.; de Crevoisier, J.; Vion-Loisel, F.; Besnard, G.; Hexemer, A.; Brown, H. R.; Kramer, E. J.; Creton, C. Nanocavitation in Carbon Black Filled Styrene-Butadiene Rubber under Tension Detected by Real Time Small Angle X-Ray Scattering. Macromolecules 2012, 45 (3), 1529-1543. 